\title{
THE SYMPATHETIC NERVOUS SYSTEM IN OBSTETRIC AND GYNAECOLOGICAL PRACTICE
}

\author{
By Albert Davis, M.D., Ch.M., F.R.C.S., M.R.C.O.G. \\ Gynaecological Surgeon, Prince of Wales', French, Dulwich and St. Giles' Hospitals, London
}

The fact that some degree of pain is accepted by most women as the normal, or periodic, accompaniment of their reproductive life is reflected in the complacency with which their medical advisers, both male and female, accept disabilities in women which in male patients would demand immediate attention. As a result an enormous amount of preventable pain is borne by these long-suffering subjects, not because it is incurable but because it is regarded as inevitable. In recent years, however, considerable attention has been directed to the possibility of alleviating this type of pain, and the demand has stimulated a considerable amount of research into the influence of the autonomic nervesupply of the genitalia on both their sensory and motor functions. This has resulted in the clarification of many hitherto unexplained phenomena and in the elaboration of several methods of neurological interference, both minor and major, in the attempt to interrupt pain-producing impulses or pain pathways. The purpose of this paper is to summarize these anatomical and physiological data, to correlate them with pathological observation and to indicate where their application to treatment might be of value in clinical practice.

\section{Anatomy and Physiology}

The uterus receives sympathetic nerves from the abdominal system and parasympathetics trom the nervi erigentes. The abdominal fibres originate in the coeliac plexus, a complex mass of fibres and ganglia arising from the confluence of the vagus and splanchnic nerves and surrounding the coeliac axis. From here strong fibres run downwards on either side of the aorta, becoming reinforced from the renal plexuses to form the intermesenteric nerves. At the level of the bifurcation of the aorta these nerves fuse to form the superior hypogastric plexus, a bundle of several strong intercommunicating filaments better known as the presacral nerve. After receiving branches from the lumbar ganglionated trunk the presacral nerve passes downwards over the fifth lumbar vertebral body and the promontory of the sacrum, below which it bends sharply backwards into the pelvis and almost immediately divides into the inferior hypogastric plexuses. These are two flat bundles of considerable length, each running at first downwards, outwards and backwards along the lateral pelvic wall, behind the internal iliac vessels just lateral to the side of the rectum and medial to the levator ani and sacral plexus. Each then enters the corresponding sacro-uterine fold, and runs forwards in it to join the nervi erigentes and form the pelvic plexus.

The nervi erigentes carry the parasympathetic supply to the uterus. They are long, fine branches arising from the anterior divisions of the middle three sacral nerves, from which they run forwards and outwards in the sacro-uterine folds, at the anterior end of which they join the hypogastric plexuses to form the great pelvic plexus or plexuso of Frankenhauser. This is a quadrilateral flat mass of neuro-fibrous tissue lying by the side of the ampulia of the rectum. It serves as a vehicle. for the admixture of the sympathetic and parasympathetic nerves which supply it, and which are by its means distributed in their correct proportion to the pelvic viscera. These receive bundles of fibres from the front of the plexus, the individual viscera being supplied by nerves which mainly accompany the vessels to the particular organ in their final distribution. In the uterus the nerves form a widely meshed plexus at the side of the body, just beneath the peritoneal coat ; from here they enter the muscularis at right angles to end in the myometrium and the capillaries. In the cervix an additional plexus is formed just beneath the squamous epithelium of the tip. The discovery of this plexus (Davis, I933) has clarified certain previously unexplained clinical results, e.g. the relief of dysmenorrhoea by cervical dilatation, the mechanism of first stage labour pain, the cause of some sudden deaths, etc. (see later).

Central connections. In the spinal column the sympathetic efferent fibres lie in the intermediolateral tract and the parasympathetics in the medioventral. The efferents run just behind and medial to the former. The central sympathetic nuclei lie in the posterior part of the hypothalamus, and the parasympathetic in its anterior portion (Beattie, 1932). The cortical centres probably lie in the 
pre-motor area close to those described for the intestine by Sheehan (1934).

The ovary is supplied by the ovarian plexus, which arises from the renal and mesenteric ganglia. It consists of two or three long communicating nerves which course downwards along the ovarian artery to the hilum, from which short branches supply all the structures of the organ. The Graafian follicles are each surrounded by a cup-like meshwork, the clinical significance of which will be discussed later.

Physiology. The nerves of the uterus are not essential for normal parturition, which can occur after complete sympathetic denervation and also after transection of the spinal cord. In normal circumstances, however, there is no doubt that they play a considerable part in the control of uterine activity. The precise action of each constituent has not yet been elucidated, but it is fairly definite that the sympathetic contracts the circular and inhibits the longitudinal muscle, while the parasympathetic exerts the opposite effect, the actions being mutually synergistic. The former also controls vascular tone and in addition carries most of the pain-sensory fibres from the organ. The ovarian nerves have similar functions.

With regard to sensation, the endometrium is insensitive to the usual stimuli. It is true that curettage of the unanaesthetized uterus is occasionally painful, and that such sensitivity is abolished by the previous intra-uterine application of cocaine, but then the operation itself is a much exaggerated stimulus. One is inclined to suggest that the pain in these cases is due to the tearing of nerve-fibres as the endometrium is stripped up, rather than to a specific and wholly unphysiological sensibility.

The peritoneal surface shares the general insensibility of the visceral peritoneum elsewhere.

The myometrium is of course sensitive to certain stimuli, namely exaggerated dilatation or contraction. A bag placed in its cavity and forcibly distended causes acute pain of both types (due to tension and spasm). The contractions of labour and the pain of artificial dilatation of the cervix are clinical examples. The ovary, though insensitive to most stimuli, is like the testis, markedly sensitive to moderate pressure, and the pain produced in this manner is strikingly similar in the two organs.

\section{Clinical Application}

To illustrate the clinical application of these data it is convenient to consider how certain specific painful pelvic conditions are affected by interference, either pathological or operative, with these autonomic pathways.

\section{Dysmenorrhoea}

The pain of spasmodic dysmenorrhoea is due to excessive contraction of the myometrium, amounting literally to a spasm, with a resultant intrauterine pressure of anything up to $180 \mathrm{~mm}$. of mercury (Moir, 1934) and subsequent vascular occlusion. The pain may be muscular or vascular in origin, but in either case is of sympathetic origin.

The great majority of primary dysmenorrhoeas are adequately relieved by carefully supervised medical treatment, including physiotherapy, specific exercises, mild analgesics, etc., but some remain intractable to these measures. These react well to various forms of sympathetic denervation, particularly when the spasmodic element predominates.

The oldest and simplest procedure is dilatation of the cervix. This is a type of sympathectomy, for it owes its effect to rupture of the sympathetic plexus which surrounds the external os. Unless this is rapidly and adequately stretched, however, the plexus remains undamaged, accounting for many of the failures following the procedure. The relief commonly observed after parturition is due to the accompanying stretching and laceration which occurs in the majority of primigravidae. To be effective, therefore, the procedure must be carried out radically and the cervix torn sufficiently? to accommodate a large bougie (it is euphemistic to talk of 'dilatation' beyond No. ro Hegar). In addition, a hollow pessary must be left in the cervical canal to prevent nerve regeneration. This somewhat barbarous procedure adds the possibility of chronic cervicitis for infection of the laceration is inevitable, and with the advent of less traumatic methods it should fall into desuetude.

Alcohol injection. The aim of this procedure (Davis, I936) is to block all the uterine nervesupply as it lies concentrated in the pelvic plexus. If the correct anatomical relations are borne in mind, the method is a relatively simple one and may be carried out under minimal intravenous anaesthesia. With the patient in the lithotomy position, the lateral fornix is exposed by opposite traction on the cervix, and the point of a long non-flexible needle inserted I $\mathrm{cm}$. lateral to the cervix. It is then passed laterally and posteriorly at an angle of $45^{\circ}$ to both the horizontal and vertical planes for $1.5 \mathrm{~cm}$., being guided to the side of the ampulla of the rectum by an internal finger. This is the situation of the pelvic plexus, and here I $\mathrm{ml}$. of absolute alcohol is slowly injected, the needle point being kept constantly moving and aspirated intermittently to avoid intravascular injection. The same procedure is then carried out on the other side. As a reinforcement a further $\mathrm{I} \mathrm{ml}$. of alcohol is then injected into the lateral 
parametrium just lateral to the supravaginal cervix, to catch any fibres which may have escaped the plexus block (Blos, 1929).

The results of this procedure are good. A follow-up of 6r personal cases showed a permanent cure-rate of 70 per cent., as against an estimated average of 20 per cent. from simple dilatation of the cervix. It is therefore the method of choice in those cases of primary spasmodic dysmenorrhoea which are resistant to ordinary medical measures.

Resection of the fresacral nerve. In patients who remain unaffected by single or repeated alcohol injection the more radical operation of presacral sympathectomy, first described by Cotte in 1923 , is indicated. Complete denervation of the uterus is unnecessary, for it is the abdominal sympathetic constituent alone which carries the contractile impulses, exaggeration of which is responsible for the spasm characteristic of dysmenorrhoea. These sympathetic fibres are concentrated in front of the fifth lumbar vertebra as the presacral nerve, and it is in this situation that they are most readily accessible to section.

Exposure of the nerve is readily attained through a short midline subumbilical incision. Good anaesthesia and the highest Trendelenberg position are essential. The intestines are packed upwards, and the sacral promontory identified. The nerve lies on the body of the fifth lumbar vertebra, in the space between the common iliac vessels. The peritoneum over this area is picked up, incised vertically for $4 \mathrm{~cm}$. and held away laterally to expose the pre-lumbar space. The plexus is now usually easily seen coursing downwards in the middle line as a single or as several communicating nerves, but as it is often adherent to the posterior parietal peritoneum this should be denuded if there is any difficulty in identifying the nerves. It is also well to pick up any lateral fibres, accommodating the whole leash in an aneurysm needle. A piece of nervous tissue $2 \mathrm{~cm}$. in length is now excised, ligature being unnecessary as bleeding is minimal, and the peritoneum is repaired with a running suture.

The operation is a relatively simple one, and careful identification of the neighbouring vessels and ureter will eliminate any danger of serious injury. Naturally any coincident pelvic pathology will be dealt with at the same time.

The results of this operation are on the whole good, and in carefully selected cases one can practically guarantee at least adequate relief. Reports by Cannon (1937), Cannaday (1938), Greenhill (r942), Cotte (1947), and many others give a high percentage of cures. Of my own series of 86 cases, 67 are free from pain over periods varying from one to 16 years. The operation should not, however, be undertaken lightly, for it carries with it the risks associated with laparotomy, and though these may be minimal, they should not be hazarded until after the failure of minor procedures. That these are reasonably successful is shown by the fact that my cases represent only 4.8 per cent. of all patients attending with the single complaint of dysmenorrhoea.

\section{Ovulalgia}

A good many women complain of brief attacks of pain in one or other side of the lower abdomen about a week after menstruation ('mittelschmerz'). The attacks are synchronous with ovulation, as shown by checking with temperature charts and ovarian palpation. When colicky in type, the pain is probably due to tubal contractions which occur at this time. The tearing pain sometimes complained of is almost certainly due to irritation of the parietal peritoneum by the follicular fluid and blood liberated on rupture. Generally, however, the pain is described as of tension type, and in these cases it almost certainly results from pressure on the peri-follicular plexus by a distended follicle. It has been shown elsewhere (Davis, 1939) how these nerves clasp the follicle like a bowl, so that when the ovary becomes cystic through failure to rupture (sclerocystic disease), chronic pain of a similar nature may result. This latter type may be exaggerated by the congestion of the period ("ovarian dysmenorrhoea,' Browne, I939).

In the majority of cases the pain of ovulalgia may be ignored, but occasionally it is severe enough to warrant ovarian sympathectomy, This consists in simple excision and ligature of a portion of the infundibulo-pelvic ligament ; separate dissection is tedious and unnecessary. The results are good in carefully selected cases, but the possibility that the denervation may interfere with maturation of the ovum in a subsequent pregnancy should always be borne in mind.

\section{Malignant Neuralgia}

The intractable pain of inoperable carcinoma of the uterus may be of either visceral or somatic origin. It is important to differentiate between the two clinically for the latter will obviously be unaffected by sympathetic block. Somatic pain is probably due to a posterior radiculitis (Todd, I934) and may be referred to the area of peripheral distribution of any of the lumbar or sacral roots. It is a severe localized neuralgia, often associated with paresis and paraesthesia. The visceral pain is due to malignant infiltration or compression of the pelvic sympathetic plexus or its branches (Bruckner and Mezinescu, 1903). This causes a severe 
generalized bearing-down pain in the pelvis, with radiation into the vulva and anal canal.

Vaginal alcohol injection is usually impracticable in these cases on account of local malignant infiltration, but presacral neurectomy will relieve purely visceral pain, and should be carried out when the general condition of the patient is sufficiently good. In debilitated subjects, and where abdominal masses are present, the method of choice is intrathecal alcohol injection. This procedure, introduced by Dogliotti (r93I) and popularized by Green (1936), is designed to destroy the sympathetic pain-afferents entering with the posterior roots. The technique is simple, and consists in the injection of $0.5 \mathrm{ml}$. of absolute alcohol without admixture into the tourth lumbar interspace, the patient lying on the side and slightly prone, and the head end of the table depressed to an angle of 15 degrees. This position is retained for an hour, and the same procedure repeated after ten days for the opposite side. The method has the advantages of simplicity and safety, and can, if necessary, be carried out in the patient's own bed. Greenhill (1947), who has an unrivalled experience of the operation, produced complete relief in more than half of his cases. Although the writer's personal experience is not so satisfactory, paralytic complications having occasionally followed, there is no doubt of the general efficacy of this type of subarachnoid injection in many cases of malignant neuralgia.

Magnesium sulphate is an efficient neurocaustic which is less generally destructive, and Bates and Judovitch (1942), have advocated its substitution for alcohol in subarachnoid injections. General experience (Bourne and Williams, 1945) has so far been disappointing, but the field is a promising one.

Todd (1937) and Kenny (1947), have used efidural procaine analgesia for the relief of malignant neuralgia with moderate success. The technique is similar to that used in obstetrics, and consists in the injection of $50 \mathrm{ml}$. of a 1.5 per cent. solution of procaine in arachis oil into the sacral canal. The method is often difficult and the pain relief usually temporary, but repeated injection is practicable in experienced hands, and undoubtedly has its application to some cases. The same remarks apply to paravertebral procaine block, originally introduced by Delliapiane and Badiano (I927), and practised more recently by Jarvis (1944) and others.

\section{General Displacements}

The lateral horizontal axis of rotation of the uterus passes through the supravaginal cervix, approximately at the level of the pelvic plexus. The organ may therefore be anteverted or retro- verted without exerting any undue nervous traction. This accords with the well-known clinical fact that 'physiological' displacement in either of these directions is normally painless. The pain of pathological retroversion is, in the majority of cases, the result not so much of the abnormal position as of its original inflammatory cause except in exaggerated cases where the cervix is pushed so far forward as to exert undue tension on the uterosacral ligaments. In prolapse, however, the uterine descent necessarily exerts considerable tension on the plexus with the resultant referred backache characteristic of the condition. In this condition a distinction must be made between simple cystocele and rectocele and true uterine prolapse. The former displacements involve merely a longitudinal traction on the long nerves passing downwards in the loose tissue between the vagina and the bladder or rectum; as a consequence both conditions may be relatively painless.

\section{Recto-vaginal Endometrioma}

The often intensely painful nature of this condition is well known, although at first sight the looseness of the cellular tissue between the vagina and the rectum appears to negative any considerable nerve traction. This is indeed true of noninfiltrating tumours and one has seen a huge recto-vaginal dermoid which remained symptomless until it produced obstruction during labour. The rapidly invasive tendency of an endometrioma, however, soon involves the nerves contained in the septum and instead of being pushed aside they are infiltrated by and enclosed within the tumour. The nerves in question are long fine filaments descending from the inferior border of the pelvic plexus. They are extremely numerous being destined for the supply of more than two-thirds of the vagina and rectum and microscopic section of the retrovaginal septum reveals their astonishing richness and complexity. Endometriomatous involvement of this rich meshwork is in itself not necessarily painful but the increased pressure accompanying the periodic menstrual enlargement of the tumour is responsible for the characteristic severe vaginal and rectal neuralgia.

Of similar aetiology is the so-called 'pelvic cry.' This is a reflex call of peculiar character which may be elicited by pressure in the pouch of Douglas in the lightly anaesthetized patient during an abdominal operation, or by similar pressure in the posterior fornix when making a pre-operative vaginal examination. It has variously been called 'Albertin's reflex' (after the surgeon who first described it), the 'Esplanade reflex' (for the extraordinary reason that the Japanese actors housed in the Esplanade during the Paris ex- 
hibition made a similar noise) and by other names, but the name 'pelvic cry' appears most appropriate. It is due, of course, to almost direct stimulation of the pelvic plexus which occupies each side of the base of the pouch and is only separated from the peritoneum by a small amount of cellular tissue.

A similar reflex is often produced by the separation of the upper part of the posterior vaginal mucosa during the performance of posterior colporrhaphy. It has been considered by some writers as identical with the 'rectal call' of anal dilatation but is really of quite different aetiology. As has been shown .above, the nerves from the pelvic plexus destined for the vagina and rectum enter the former from behind and the latter from in front. They are concentrated, therefore, in the rectovaginal septum, particularly in its upper part, and it is the operative stimulation of this extremely rich nervous meshwork which causes the reflex laryngeal spasm.

\section{Pericervicitis}

The cervix, like the uterus, is normally insensitive to movement. Violent displacement of either organ during examination or coitus is, of course, uncomforable but actual pain is caused only if the movement is predominantly vertical, for the position of the pelvic plexus at the fixed junction protects its branches from traction except in this axis. In inflammatory diseases of the pericervical tissues, however, painful movement of the cervix may be a striking clinical phenomenon; it is also the cause of the severe dyspareunia often experienced by these patients. The underlying condition is an inflammatory infiltration in the lateral cervical parametrium and the uterosacral ligaments, resulting as a rule from endocervicitis, and the symptoms are rapidly relieved by paracervica! alcohol injection when the acute phase has subsided.

Young's syndrome. James Young has recently drawn attention to the frequent association of iliac fossa pain with chronic cervicitis. Both are, of course, common gynaecological disorders but the fact that they may be aetiologically connected has hitherto escaped observation. Clinically the sundrome manifests itself in chronic lower abdominal pain, dyspareunia and leucorrhoea, with no obvious pelvic pathology except cervicitis. The pain in the iliac fossa may be evoked by movement of the cervix which is itself often exquisitely tender. The pain is resistant to all the routine methods of treatment including major operative procedures, but it reacts immediately to protocaine injection of the pelvic plexus. Young explains this phenomenon by suggesting that the hypogastric pain is a true visceral pain, the iliac fossa pain being referred from the cervix to the abdominal area of its peripheral parietal segmental representation, the iliac fossa. This application of the Head-Mackenzie theory of visceral sensibility to the cervix is at once ingenious and clinically satisfactory, and though exception may be taken on anatomical grounds to the implication that the cervical innervation is exclusively autonomic, there is no doubt that Young's hypothesis adequately explains many hitherto difficult cases.

\section{Posterior Parametritis}

In this condition, the clinical features of which I have called the ' posterior parametritis syndrome,' the lower part of the pelvic plexus and its adjacent nerves are involved in a diffuse lymphangitis as they lie within the uterosacral folds. The primary cause is a severe chronic cervicitis, and as a result of the constant inflammatory process the secondary neuritis is similarly constant. The patient thus complains of continuous low backache, aggravated by menstruation and coitus. The uterosacral ligaments are readily palpated from the vagina as hard, tender, projecting cords with the uterus pulled into anteversion by their secondary contracture. Many French authors, notably Molin (1929), believe the condition to be due to the unsatisfied spasm of coitus interruptus, but though this practice may aggravate the disorder, there can be little doubt of its primary inflammatory origin. Novocaine injection of the utero-sacral ligaments is a satisfactory treatment for this syndrome. The method is a simple one and consists in the injection of $\mathrm{I} \mathrm{ml}$. of 2 per cent. novocaine into the most anterior identifiable extremity of each ligament. Anaesthesia is unnecessary and though the initial relief is often only temporary, the procedure is easily repeated. In intractable cases, absolute alcohol may be substituted, though here the total quantity used is halved, and the injection placed superficially and medially to avoid ureteric and uterine vessel damage.

\section{Ectopic Gestation}

The pain in unruptured tubal gestation is due to local tension on the circular submucous plexus caused by rapid enlargement within the lumen. It is continuous, fairly severe and neuralgic in character, with interspersed colic resulting from reflex tubal contraction.

When an ectopic gestation ruptures, the pain is equally characteristic. It occurs in attacks lasting from a few to several minutes and is 'tearing' in character. The early attacks are due to the initial projection of blood on to the sensitive peritoneum and later to involvement of fresh areas by bodily movements or further haemorrhage. The pain is precisely the same as that of perforated peptic 
ulcer. Graafian follicle haemorrhage or any other condition causing acutera extvasation on to a hitherto normal peritoneal surface is referred to the area of peripheral distribution of the particular spinal segments affected. These vary considerably and although the pain is usually situated in the lower abdomen commencing in the iliac fossa of the affected side, it may extend considerably higher, and I recently saw a case in which the pain was so exclusively epigastric that a diagnosis of perforated gastric ulcer was made and acted upon by a general surgeon. The shoulder tip pain so often present is probably due to diaphragmatic irritation, both the phrenic nerve and the cutaneous nerves of the shoulder being derived from the third, fourth and fifth cervical segments. Of additional interest is the manner in which in small haemorrhages, and in the early stages of larger ones, the pain is localized to the shoulder of the same side as the rupture. Scheumann is sceptical of the accuracy of this fact, but with Rubin and others I have found it correct in the majority of cases.

It is important to remember that small haemorrhages into the tubal wall may closely simulate the pain of tubal rupture or tubal abortion, and in a patient operated upon recently under this diagnosis there was no free blood whatever, a distended but unruptured isthmic gestation being present. The pain in this case occurred in spasms, described as cutting in character, but there was no distension pain or colic. A further point is that the first symptom in many cases of ectopic gestation is the actual rupture, though close subsequent enquiry will often reveal the presence of some previous discomfort often so slight as to be almost unnoticeable. Variations in individual sensibility, together with differences in the rapidity of the tubal distention would appear to account for this discrepancy.

\section{Labour Pains}

The neurogenic basis of the pains of labour is somewhat complicated. The pain is actually felt in the uterus itself in only a small number of cases. In the majority it is referred to the peripheral area of distribution of the last two dorsal, the first lumbar and the middle three sacral nerves. It is thus felt mainly in the deeper parietes of the lumbar, sacral, inguinal and hypogastric regions, and is accompanied by definite modifications in cutaneous sensibility (hyperaesthesia, hyperalgesia). This reference is further proved by the fact that the subcutaneous novocaine injection of the appropriate areas often relieves the pain.

In spite of this, however, the peripheral cerebrospinal nerves involved are not themselves directly affected by pressure from the uterus, as has often been suggested. Such direct pressure is anatomically impossible, for the projection of the lumbar vertebrae effectively prevents simultaneous bilateral pressure on the nerves of the lumbar plexus, while the sacral plexus, as Aburel points out, is well protected by tautly stretched ligaments. One may therefore postulate a primary stimulation of the pelvic plexus from the myometrial nerveendings which are themselves directly stimulated by the chemical accumulation of muscular hypercontraction, centripetal transmission taking place along the autonomic nerve-pathways described above and overflow in the spinal cord to the adjacent posterior root ganglia resulting in reference along the posterior roots to the peripheral areas of supply of the particular cerebrospinal nerves affected.

\section{Phlegmasia Alba Dolens}

Apart from the severe and often intractable pain associated with this condition, the permanent oedema and disability which so often follow give its appearance in the young parturient a particular significance. The pain and much of the subsequent vascular damage are due to arterial spasm, reflex from the acute venous thrombophlebitis. To counteract this Leriche (1934) carried out lumbab ganglionic block (the sympathetic being pree dominantly vaso-constrictor). The technique consists in the injection of $5 \mathrm{cc}$. of a I per cent procaine hydrochloride solution into and around the lumbar sympathetic cord. With the patient lying horizontally and on the side, the needle is inserted at a point $3 \mathrm{~cm}$. lateral to the spinous process, and passed until the transverse process is reached. It is then moved medially and passed a further $3 \mathrm{~cm}$. along the lateral border of the vertebra where it reaches the ganglionated cord. The injection is made after preliminary aspiration. My own experience of this method is restricted and the results equivocal, but there is no doubt of the dramatic improvement when the injection is successful, the intense pain immediately disappearing, and the oedema rapidly diminishing.

\section{Summary}

An endeavour has been made to show that most types of pelvic pain in women have a relatively simple neurological basis. The pain may be due to direct traction on nerve trunks, to irritation of nerve endings or to malignant or inflammatory infiltration of nerve plexuses. The frequency with which microscopic abscesses are found in presacral nerves removed for intractable pelvic pain has been shown elsewhere (Davis, 1938). It is, of course, obvious that the prime factor in relieving pain is the removal or other treatment of any obvious gross pathology, but there remains a 
considerable number of patients in whom clinical changes are not obvious. In these cases appropriate neurosurgery, from alcohol injection to section, is of considerable value. Such measures are held also to be a valuable adjunct to general pelvic surgery in the eradication of long-standing and previously intractable pain.

\section{BIBLIOGRAPHY}

BATES, W., and JUDOVITCH, B., Anaesthesiology, 3, 663. BEATTIE, J. (1932), F. Canad. Med. Ass., 26, 400. BROWNE, O'D. (1939), Irish F. Med. Sc., 65, 73. BLOS, D. (1929), Munch. Med. Wchnschr., 76, 1173. BRUCKNER and MEZINES, C. V. (1903).

CANNADAY, J. E. (1938), Trans. Int. Coll. Surg., I, 160.
CANNON, D. J. (1937), f. Obst. and Gyn., Brit. Emp., 44, 13. COTTE, G. (1925), Lyon Med., 135, 153.

DAVIS, A. (1933), Ұ. Obst. and Gyn., Brit. Emp., 40, $48 \mathrm{r}$.

DAVIS, A (1936), Lancet, $x, 80$.

DAVIS, A. (1939), F. Obst. and Gyn., Brit. Emp., 46, 962.

DAVIS, A. (1938), 'Dysmenorrhoea,' Oxf. Univ. Press, London, p. I2I.

DELLIAPINE, G., and BADIANO, P., Clin. ostet. sinec., 29, 537

DOGLIOTTI, A. M. (1931), Presse Med., 39, 1249.

GREENHILL, J. P., and SCHMITZ, H. E. (1936), Am. F. Obst. and Gyn., 31, 290.

GREENHILL, J. P. (1947), Brit. Med. Y., 2, 859.

GREENHILL, J. P. (1942), Year Book of Obst. and Gyn., p. 543.

JARVIS, S. M. (1944), Am. F. Obst. and Gyn., 47, 335.

KENNY, M. (1947), Brit. Med. F., 2, 862.

LERICHE, R. (1934), Presse Med., 42, 1481.

MOIR, C. (1934), Trans. Edin. Obst. Soc., 54, 93.

SHEEHAN D (1934), f. Physiol., 83, 177.

TODD, T. F. (1937), Lancet, 2, 555 .

\section{$\star \quad$ NEW HEINEMANN BOOKS 1949}

\section{OCCUPATIONAL EYE DISEASES}

By JOSEPH MINTON, M.D., F.R.C.S.

Deals with the injuries that can occur to the eye in factories and workshops. Of special interest to doctors in the industrial medicine service, surgeons, and medical officers of health.

Demy 8vo. 176 pages. 30 illustrations, ro coloured plates. (Ready approx. August.) 17s. 6d.

THE COMPARATIVE ANATOMY AND PHYSIOLOGY

OF THE LARYNX

By V. E. NEGUS, M.S., F.R.C.S. (author of Mechanism of the Larynx)

The subject is divided into sections connected with the numerous functions of the larynx. The original specimens from which the majority of the illustrations are derived are in the Museum of the Royal College of Surgeons of England.

Med. 8vo. 204 pages. 190 illustrations.

(Ready approx. Fuly.) 3 os.

\section{THE CONQUEST OF MALARIA}

\section{By JAIME JARAMILLO-ARANGO}

A unique history tracing the treatment of malaria from quinine to the latest drug paludrine, based on South American, Spanish, Portuguese, and Italian documentary sources never before available. Written by a distinguished Central American physician with a world-wide outlook. Of special interest to tropical disease experts, botanists, historians.

NEW EDITIONS :

$$
\text { Demy 8vo. } 136 \text { pages. } 20 \text { illustrations. (In preparation.) 16́s. }
$$

POSITIONING IN RADIOGRAPHY. 5th edition by K. C. ClaRK. 75 s.

COMPLETE OUTLINE OF FRACTURES. 3rd edition by J. GRANT BONNIN. $30 s$.

STERNAL PUNCTURE. 4th edition by ALFRED PINEY and J. L. HAMilton-Paterson. 15.

SOCIAL BIOLOGY. 2nd edition by Alan Dale. 15 .

THE RHESUS FACTOR. 2nd edition by G. FULTON ROBERTS. 3s. $6 d$.

TEXT-BOOK OF GYNAECOLOGY. 3rd edition by J. H. PerL. 22s. $6 d$. 